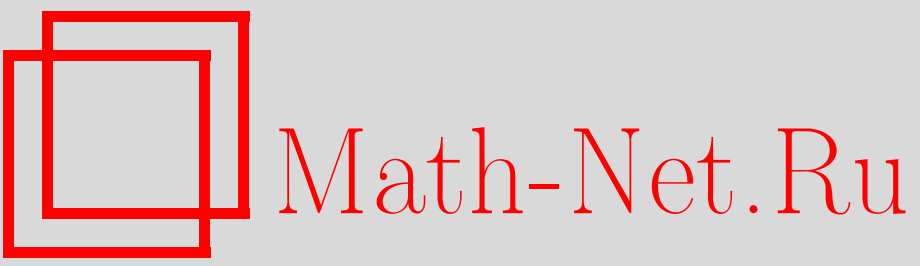

А. А. Славнов, Мягкое нарушение суперсимметрии как спонтанное нарушение во вспомогательном секторе, ТМФ, 1998, том 117, номер 2, 325-329

DOI: https://doi.org/10.4213/tmf934

Использование Общероссийского математического портала Math-Net.Ru подразумевает, что вы прочитали и согласны с пользовательским соглашением

http://www . mathnet.ru/rus/agreement

Параметры загрузки:

IP : 107.22 .136 .117

26 апреля 2023 г., 06:59:45 
ТЕОРЕТИЧЕСКАЯ

И МАТЕМАТИЧЕСКАЯ

ФИЗИКА

Том 117, № 2

ноябрь, 1998

(C) $1998 \mathrm{r}$.

А. А. Славнов*

\section{МЯГКОЕ НАРУШЕНИЕ СУПЕРСИММЕТРИИ КАК СПОНТАННОЕ НАРУШЕНИЕ ВО ВСПОМОГАТЕЛЬНОМ СЕКТОРЕ}

Мягкое нарушение суперсимметрии реализовано как спонтанное нарушение за счет расширения сектора вспомогательных полей. Обсуждается процедура перенормировки в подобных моделях.

\section{1. ВВЕДЕНИЕ}

Для применения суперсимметрии (SUSY) к реальному миру необходим какой-либо механизм нарушения SUSY. B то же время желательно максимально сохранить такие важные предсказания SUSY-теорий, как теорема о неперенормировке. Желательно также сохранить возможность использования удобных технических средств, сушествуюших в SUSY-моделях, в частности супердиаграммной техники. С этой точки зрения наиболее привлекательным является спонтанное нарушение SUSY, однако этот механизм оказывается слишком ограничительным и не позволяет конструировать реалистические модели.

На практике вводятся так называемые мягкие нарушаюшие симметрию члены, как, например, массовые члены и некоторые тройные скалярные вершины. Недавно появился ряд публикаций, в которых обсуждается процедура перенормировки в моделях с мягко нарушенной SUSY $[1-3]$ в рамках так называемого "шпурионного" механизма мягкого нарушения SUSY [4-7].

В данной работе будет показано, что этот механизм в действительности является специальной реализацией обшего метода спонтанного нарушения SUSY во вспомогательном секторе, развитого ранее в серии работ, посвященных суперсимметричным калибровочным теориям [8-11]. В этих работах было показано, что мягкое нарушение SUSY можно реализовать как спонтанное нарушение, если расширить модель, вводя дополнительные вспомогательные суперполя, которые в конечном итоге отшепляются от физического сектора. Эта формулировка позволяет сохранить наиболее важные предсказания SUSY-теорий, такие как обобшенные SUSY-тождества Уорда [12-14].

* Математический институт им. В. А. Стеклова РАН, Москва, Россия. E-mail:slavnov@mi.ras.ru 
Ниже будет показано, что некоторые соотношения между константами перенормировки жестких и мягко нарушенных SUSY-моделей, которые были получены в работах $[2,3]$, можно получить простым способом из нашего формализма.

\section{2. РАСШИРЕННЫЕ SUSY-МОДЕЛИ СО СПОНТАННО НАРУШЕННОЙ СИММЕТРИЕЙ}

Идея метода состоит в следуюшем. Суперсимметричный калибровочно-инвариантньй лагранжиан можно записать в виде

$$
L=\int d^{2} \bar{\theta} d^{2} \theta L_{\mathrm{g}}+\int d^{2} \bar{\theta} L_{\mathrm{ch}}^{-}+\int d^{2} \theta L_{\mathrm{ch}}
$$

Здесь $L_{\mathrm{g}}$ - суперполе общего вида, а $L_{\mathrm{ch}}$ - киральное суперполе. Например, в теории Янга-Миллса $L_{\mathrm{g}}=0$,

$$
L_{\mathrm{ch}}=-\frac{1}{8 g^{2}} \operatorname{Tr} W^{\alpha} W_{\alpha}
$$

Здесь $W^{\alpha}-$ киральный тензор напряженности,

$$
W^{\alpha}=\bar{D}^{2}\left(e^{-V} D^{\alpha} e^{V}\right)
$$

$V=V^{A} T^{A}, V^{A}$ - калибровочное суперполе и $T^{A}$ - генераторы калибровочной групшы, $D$ - суперковариантная производная.

Введем вспомогательные суперполя $P, P^{\prime}, R_{+}, R_{+}^{\prime}$, где $P, P^{\prime}$ - суперполя общего вида, а $R_{+}, R_{+}^{\prime}$ - киральные суперполя. Все эти поля являются синглетами калибровочной группы. Тогда можно написать новьй суперсимметричный и калибровочно-инвариантный лагранжиан следующего вида:

$$
\begin{aligned}
L^{\prime}= & \int d^{2} \bar{\theta} d^{2} \theta\left[L_{\mathrm{g}}(1+P)+P_{+}^{\prime} \square P_{-}+P_{-}^{\prime} \square P_{+}+P_{1}^{\prime} \square P_{1}+\kappa P^{\prime}+R_{+}^{\prime} R_{-}+R_{+} R_{-}^{\prime}\right]+ \\
& +\int d^{2} \theta\left[L_{\mathrm{ch}}\left(1+R_{+}\right)-\mu R_{+}^{\prime}+\text { h.c. }\right] .
\end{aligned}
$$

Здесь $P_{+}, P_{-}, P_{1}$ обозначают неприводимые компоненты суперполя $P, P=P_{+}+$ $P_{-}+P_{1}$

Этот лагранжиан очевидным образом суперсимметричен и калибровочно-инвариантен. Благодаря присутствию линейных членов SUSY спонтанно нарушена: сдвигая поля $P, R_{+}, R_{-}$к стабильным значениям, мы получим массовые члены для скалярных и спинорных компонент полей, входяших в исходный лагранжиан (1), а также тройные вершины взаимодействия скалярных полей. Квадратичная форма полей $P, R_{+}, R_{-}$выбрана таким образом, что после сдвига они полностью отщепляются от физического сектора благодаря специальному виду соответствующих пропагаторов,

$$
P P=P^{\prime} P^{\prime}=R_{ \pm} R_{ \pm}=R_{ \pm}^{\prime} R_{ \pm}^{\prime}=0
$$


Единственный наблюдаемый эффект, производимый этими полями, состоит в появлении членов, мягко нарушаюших SUSY. В этой схеме легко узнать “шпурионный” механизм. В самом деле, подставляя в (4) решения уравнений движения для полей $P, R_{ \pm}$, получим

$$
L \longrightarrow \int d^{2} \bar{\theta} d^{2} \theta L_{\mathrm{g}}\left(1+\kappa(\bar{\theta} \theta)^{2}\right)+\int d^{2} \theta L_{\mathrm{ch}}(1+\mu \theta \theta)+\text { h.c. }
$$

Однако удобнее работать с исходным выражением (4). Благодаря явной суперсимметрии и калибровочной инвариантности этого лагранжиана можно непосредственно использовать результаты, полученные для SUSY-моделей. В частности, как будет показано ниже, это позволяет получить некоторые соотношения между контрчленами жесткой и мягко нарушенной моделей очень простым способом.

Рассмотрим в качестве примера теорию Янга-Миллса. Мягкое нарушение SUSY можно реализовать как спонтанное нарушение в системе, описываемой следующим явно суперсимметричным действием:

$$
\begin{aligned}
A= & \int d^{4} x d^{2} \theta\left[-\frac{1}{8 g^{2}}\left(1+R_{+}\right) \operatorname{Tr} W^{\alpha} W_{\alpha}+\text { h.c. }\right]+ \\
& +\int d^{4} x d^{2} \bar{\theta} d^{2} \theta R_{+} R_{-}^{\prime}-\mu \int d^{4} x d^{2} \theta R_{-}^{\prime}+\text { h.c. }
\end{aligned}
$$

В самом деле, переходя к стабильному минимуму путем сдвига $F$-компонент полей $R_{+}, R_{-}$,

$$
\left(R_{+}\right)_{F} \longrightarrow\left(R_{+}\right)_{F}+\mu, \quad\left(R_{-}\right)_{F} \longrightarrow\left(R_{-}\right)_{F}+\mu,
$$

мы получим массовые члены для фермионных компонент суперпотенциала $W$

$$
L_{\mathrm{m}}=-\frac{\mu}{2 g^{2}}\left(\lambda^{T} C \lambda+\bar{\lambda} C \bar{\lambda}^{T}\right)
$$

где $C$ - матрица зарядового сопряжения. После такого сдвига вспомогательные поля $R_{ \pm}, R_{ \pm}^{\prime}$ отшепляются от физического сектора, и мы получаем обычную SUSY-теорию Янга-Миллса с мягким массовым членом.

Рассмотрим структуру возможных контрчленов. Производящий функционал функции Грина дается следуюшим интегралом:

$$
Z=\int \exp i\left\{A+A_{\text {g.f. }}+A_{\text {gh }}+\int d^{2} \bar{\theta} d^{2} \theta d^{4} x g^{-1} V^{a} J^{a}\right\} d V d R
$$

где $d R$ обозначает произведение дифференциалов $R_{ \pm}$-полей. Для простоты мы полагаем, что выбрана SUSY-калибровка Лоренца, $A_{\text {g.f. и }} A_{\text {gh }}$ обозначают соответственно члены, фиксирующие калибровку, и лагранжиан духов Фаддеева-Попова.

Интегрирование по калибровочным полям приводит к появлению ультраффиолетово расходящихся диаграмм, требующих введения соответствующих контрчленов. Благодаря явной суперсимметрии и калибровочной инвариантности действия (10) единственная допустимая локальная структура имеет вид

$$
\left(\operatorname{Tr} W^{\alpha} W_{\alpha}\right) K+\text { h.c. . }
$$


Здесь $K$ - некоторая функция суперполей

$$
g^{-2}\left(1+R_{+}\right)
$$

и их ковариантных производных. По соображениям размерности члены с ковариантными производными отсутствуют. Эту функцию можно разложить в ряд по $g^{2}$, причем коэффициенты этого разложения определяются жесткой теорией. В случае жесткой SUSY $\left(R_{+}=0\right)$ соответствуюшая функция приводит к перенормировке заряда

$$
g^{2} \longrightarrow Z\left(g^{2}\right) g^{2}=g^{2}\left(1+z_{2} g^{2}+z_{4} g^{4}+\cdots\right)
$$

В нашем случае после интегрирования по $R_{+}^{\prime}, R_{-}^{\prime}$ получаем

$$
\left(1+R_{+}\right) \longrightarrow 1+\mu \theta \theta, \quad\left(1+R_{-}\right) \longrightarrow 1+\mu \bar{\theta} \bar{\theta}
$$

В уравнении (7) эти функции интегрируются по $d^{2} \theta$ и $d^{2} \bar{\theta}$, соответственно. Поэтому их можно записать в симметричном виде

$$
1+\mu \theta^{2}+\mu \bar{\theta}^{2}=\left(1-\mu \theta^{2}-\mu \bar{\theta}^{2}+2 \mu^{2} \theta^{2} \bar{\theta}^{2}\right)^{-1}
$$

Полином по $g^{2}$ приобретает вид

$$
\begin{aligned}
& 1+z_{2} g^{2}\left(1-\mu \theta^{2}-\mu \bar{\theta}^{2}+2 \mu^{2} \bar{\theta}^{2} \theta^{2}\right)+\cdots=Z\left(g^{2}\right) \\
& g^{\prime 2}=g^{2}\left(1-\mu \theta^{2}-\mu \bar{\theta}^{2}+2 \mu^{2} \bar{\theta}^{2} \theta^{2}\right) .
\end{aligned}
$$

Следовательно, в мягко нарушенной теории константа $g^{2}$ жесткой теории должна быть заменена на $g^{\prime 2}$. Это согласуется с результатом работы [2]. Заметим, что если бы мы рассматривали калибровку более общего вида, нужно было бы ввести специальным образом фиксируюшие калибровку члены, чтобы обеспечить зависимость ультрафиолетовых контрчленов только от $R_{+}, R_{-}$. Эта проблема обсуждается в работе [2].

Чтобы устранить расходимости функции Грина в жесткой теории, нужно еще перенормировать суперполе $V$,

$$
V \longrightarrow Z_{V}^{1 / 2}\left(g^{2}\right) V
$$

Аналогичные рассуждения показывают, что в рассматриваемой модели соответствующая перенормировка обеспечивается путем замены $g^{2} \rightarrow g^{\prime 2}$. В действительности, если мы интересуемся только “физическими” функциями Грина (включающими $A_{\mu^{-}}, \lambda$ - и $D$-компоненты суперполя $V$ ), то эта замена несущественна и можно пользоваться жесткой константой перенормировки $Z_{V}\left(g^{2}\right)$. 


\section{3. ЗАКЛЮЧЕНИЕ}

Аналогичный, хотя и более сложный анализ можно провести в том случае, когда включены все возможные типы спонтанного нарушения SUSY, описанные вьше. Заметим, что наш метод не приводит к появлению массовых членов для фермионных компонент суперполей материи, т.к., в отличие от массовых членов для гравитино, эти члены не являются мягкими. Чтобы получить более детальные предсказания, в частности соотношения между конечными частями функций Грина в жесткой и мягко нарушенной теориях, нужно использовать обобщенные тождества Уорда, которые можно записать в этом подходе (см. [9]).

Эта работа поддержана грантом РФФИ № 96-01-00551 и Президентским грантом поддержки ведуших научных школ.

\section{Список литературы}

[1] Y. Yamada. Phys. Rev. D. 1994. V. 50. P. 3537.

[2] L. V. Avdeev, D. I. Kazakov, I. N. Kondrashuk. Nucl. Phys. B. 1998. V. 510. P. 289.

[3] I. Jack, D. R. T. Jones. The Gaugino $\beta$-function. hep-ph/9709364.

[4] L. Girardello, M. I. Grisaru. Nucl. Phys. B. 1982. V. 194. P. 65.

[5] J. A. Helayel-Neto. Phys. Lett. B. 1984. V. 135. P. 78.

[6] F. Feruglio, J. A. Helauel-Neto, F. Legovini. Nucl. Phys. B. 1985. V. 249. P. 533.

[7] M. Scholl. Z. Phys. C. 1985. V. 28. P. 545.

[8] A. A. Slavnov. Nucl. Phys. B. 1977. V. 124. P. 301.

[9] А. А. Славнов. ТМФ. 1976. Т. 27. № 2. С. 141; Т. 29. № 2. С. 154.

[10] A. A. Slavnov. Gauge and supergauge theories. In: Proc. of 18 International conference on High Energy Physics. Dubna: JINR, 1977.

[11] E. Sh. Egorian, A. A. Slavnov. Ann. of Phys. 1978. V. 116. P. 358.

[12] А. А. Славнов. ТМФ. 1975. Т. 23. № 1. С. 3.

[13] A. A. Slavnov. Nucl. Phys. B. 1975. V. 96. P. 134.

[14] В.К. Кривощеков, А. А. Славнов, Б. А. Файзуллаев. ТМФ. 1976. Т. 26. № 2. С. 147. 